\title{
Cooperative Effects in the Detection of a Nitroaliphatic Liquid Explosive and an Explosive Taggant in the Vapor Phase by Calix[4]arene-Based Carbazole-Containing Conjugated Polymers
}

\author{
Patrícia D. Barata and José V. Prata*[a]
}

Two fluorescent molecular receptor based conjugated polymers were used in the detection of a nitroaliphatic liquid explosive (nitromethane) and an explosive taggant (2,3-dimethyl2,3-dinitrobutane) in the vapor phase. Results have shown that thin films of both polymers display remarkably high sensitivity and selectivity toward these analytes. Very fast, reproducible, and reversible responses were found. The unique behavior of these supramolecular host systems is ascribed to cooperativity effects developed between the calix[4]arene hosts and the phenylene ethynylene-carbazolylene main chains. The calix[4]- arene hosts create a plethora of host-guest binding sites along the polymer backbone, either in their bowl-shaped cavities or between the outer walls of the cavity, to direct guests to the area of the transduction centers (main chain) at which favorable photoinduced electron transfer to the guest molecules occurs and leads to the observed fluorescence quenching. The high tridimensional porous nature of the polymers imparted by the bis-calixarene moieties concomitantly allows fast diffusion of guest molecules into the polymer thin films.

\section{Introduction}

There is increasing international concern about the intentional misuse of explosive materials for criminal purposes. The development of chemical sensors, and devices thereof, based on optical transduction processes for the detection of trace amounts of compounds found in explosives compositions has been pursued very intensively for more than a decade given their potential usefulness in enhancing the security of nations. ${ }^{[1-5]}$

Precursor chemicals to homemade explosives include the liquid explosive nitromethane (NM), which, combined with appropriate sensitizing compounds (mainly aliphatic amines, ammonia) ${ }_{1}^{[6-8]}$ has been implicated in several bombing events. ${ }^{[9]}$ Under certain conditions, sensitized NM can detonate with blasting caps to deliver more power ( $>20 \%$ ) in comparison to trinitrotoluene (TNT). ${ }^{[7]}$

Contrary to that of nitroaromatics, literature reports concerning the fluorescence detection of NM are scarce, particularly in the vapor phase. Reasons for this include less favorable photoinduced electron transfer (PET) from a given fluorophore excited state to the LUMO of NM. In addition to lower electron affinity (compared with nitroaromatics), the lack of aromatic

\footnotetext{
[a] P. D. Barata, Prof. J. V. Prata

Laboratório de Química Orgânica

Departamento de Engenharia Química

and Centro de Investigação de Engenharia

Química e Biotecnologia

Instituto Superior de Engenharia de Lisboa

Instituto Politécnico de Lisboa

R. Conselheiro Emídio Navarro, 1959-007 Lisboa (Portugal)

E-mail:jvprata@deq.isel.ipl.pt

Supporting information for this article is available on the WWW under http://dx.doi.org/10.1002/cplu.201300280.
}

rings in its structure, which are known to be involved in the establishment of $\pi-\pi$ charge-transfer complexes between the (excited) fluorophore (donor) and the (ground-state) analyte (acceptor), add further difficulties to its detection. The sensing of NM vapors represents an even greater challenge owing to its high volatility. Most studies reported so far were performed in the solution phase, either as isolated fluorophores ${ }^{[10-12]}$ or integrated in polymeric materials. ${ }^{[13-16]}$ The detection of NM in the vapor phase was only recently considered. ${ }^{[17]}$ By using a 2,7-linked carbazole trimer in the form of nanofibrils, up to $55 \%$ fluorescence quenching was achieved in a sealed-cell setup at RT. A negligible fluorescence quenching effect was found in another recent study that used a microporous metalorganic framework (MOF) as a sensory material. ${ }^{[18]}$ Most recently, disubstituted 3,6-carbazoles, possessing terphenyl moieties either directly attached to or bridged through an ethynylic linker to the central carbazole unit, were tested in the detection of NM. ${ }^{[19]}$ In the vapor phase, using a transient measurement setup, $15-25 \%$ quenching efficiency was obtained upon exposure to 2000 ppm of NM.

Many high explosives, particularly those found in plastic explosives, do not produce a significant vapor pressure that would enable their detection. For this reason, four detection taggants (2-nitrotoluene, 4-nitrotoluene, 2,3-dimethyl-2,3-dinitrobutane (DMNB), and ethylene glycol dinitrate) were select$\mathrm{ed}^{[20]}$ for inclusion in the International Civil Aviation Organization Convention, ${ }^{[21]}$ of which DMNB is most used. The DMNB structure is not well suited for interaction with planar $\pi$ systems normally found in fluorophore structures; thus limiting the overall binding and charge transfer between the excited 
fluorophore and DMNB. Possibly for this reason, and similarly to NM, studies concerning the detection of DMNB in the vapor phase have not widely been reported.

The rational design of several poly(phenylene)s containing fluorene-carbazole and fluorene-phenylene derivatives led to the first report on vapor-phase DMNB detection, ${ }^{[22]}$ in which a $20 \%$ reduction in fluorescence was reached upon exposure to equilibrium vapor pressures of DMNB. A high fluorescence quenching response $(73 \%)$ upon exposure for 2 min to saturated vapor of DMNB was later achieved by a fluorescent nanofibril made up from a cyclic tetramer of 3,6-carbazolylene-ethynylene units by using a $90 \mathrm{~nm}$ thick film in a sealed-cell setup. ${ }^{[23]}$ By using powder layers $(5 \mu \mathrm{m}$ thickness; $10 \mathrm{~s}$ exposure) of a luminescent microporous MOF ( $\left[\mathrm{Zn}_{2}\left(4,4^{\prime}\right.\right.$-biphenyldicarboxylate $)_{2}(1,2$-bipyridylethene)]), a high quenching efficiency $(84 \%)$ of DMNB vapors was reported. ${ }^{[24]}$ However, benzene and $\mathrm{N}, \mathrm{N}$-dimethylformamide (DMF) also quench the fluorescence of this MOF to a very significant extent ( 45 and $58 \%$, respectively). Another MOF ([ $\mathrm{Zn}_{2}\left(4,4^{\prime} \text {-oxybis(benzoic acid) }\right)_{2}\left(4,4^{\prime}\right.$ bipyridine)]) was later tested ${ }^{[18]}$ and had a negligible quenching effect in the presence of DMNB. Different generations of 3,6carbazole dendrimers with spirofluorene cores were recently tested as sensing materials for DMNB. ${ }^{[25]}$ Vapor detection by means of $25 \mathrm{~nm}$ thick films in a sealed-cell setup gave only moderate quenching efficiency (ca. $10 \%$ after $10 \mathrm{~s}$, reaching ca. $25 \%$ over 5 min exposure). 3,6-Carbazole-terphenyl compounds represent the most recent entries of sensing materials for DMNB. ${ }^{[19]}$ The fluorescence of thin films $(\approx 50 \mathrm{~nm})$ was quenched to an extent of only $3 \%$ at a DMNB concentration of $1.5 \mathrm{ppm}$ (roughly $6 \%$ efficiency at saturated vapor concentration, ca. $2.7 \mathrm{ppm}$ ), although in solution one of the highest ${ }^{[26]}$ quenching constants reported to date was found. Other reports also appeared and presented varying amounts of fluorescence quenching in solution. ${ }^{[14,26-28]}$

We recently synthesized phenylene-alt-ethynylene-3,6- and 2,7-carbazolylene polymers that contained calix[4]arene units (Scheme 1 a). ${ }^{[29]}$ These prototype molecular receptor based conjugated polymers (CPs) were designed for the fluorescence sensing of a wide range of analytes, in particular, explosive materials. The concept behind the assembly of calix[4]arenes with carbazole-containing CPs was based on the expected cooperativity of such systems to enhance the signaling events related to a particular host-guest interaction. We thought that, on the one hand, the good donor ability of the carbazole moieties integrated in the polymer backbone (transduction site) would enhance the sensing capabilities of electron-deficient analytes (such as nitro explosives), especially those with reduced electron affinities, and enable more exergonic electron-transfer processes to occur. On the other hand, the presence of bis-calix[4]arene components along the CP would create additional binding sites for particular guests, either in the cup-shaped cavities of the cyclic oligomers or between the calixarene units, and simultaneously would help to construct adequate porous structures for rapid and smooth vapor diffusion of a given analyte through polymer thin films.

This contribution critically tests the above hypotheses by using two hard-to-detect nitroaliphatics: NM and DMNB (Scheme 1 b). Five sensory materials were studied: two CALIXPPE-CBZs (Scheme $1 \mathrm{a})$, which differed in the structural arrangement of the diethynyl substitution (3,6- or 2,7-) pattern on the carbazole units; two non-calixarene-based polymers, TBP-PPECBZs (Scheme 1c), with the same polymer backbone; and a CALIX-p-PPE, ${ }^{[30]}$ which had a $p$-phenylene ethynylene-type main chain (Scheme $1 \mathrm{~d}$ ).

\section{Results and Discussion}

Molecules with convergent concave surfaces, such as calix[4]arenes, in the cone conformation are known as receptors (hosts) for neutral organic substrates (guests/analytes). The efficiency of a recognition event is based on the structural complementarity of host and guest: its strength is controlled by the nature of the guest, which should normally contain acidic $\mathrm{CH}$ groups to allow stabilizing $\mathrm{CH}-\pi$ interactions with the aryl re- a)

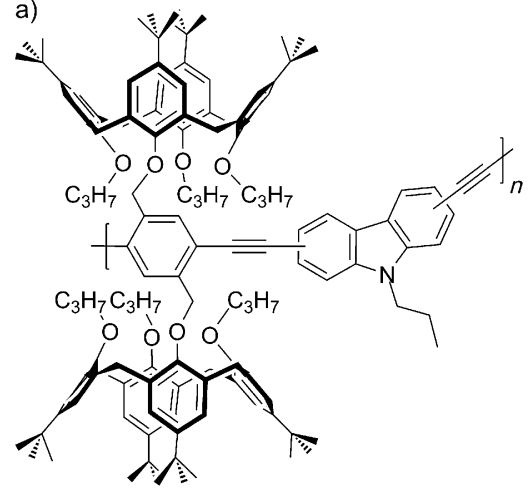

CALIX-PPE-3,6-CBZ: 3,6-linkage on CBZ CALIX-PPE-2,7-CBZ: 2,7-linkage on CBZ

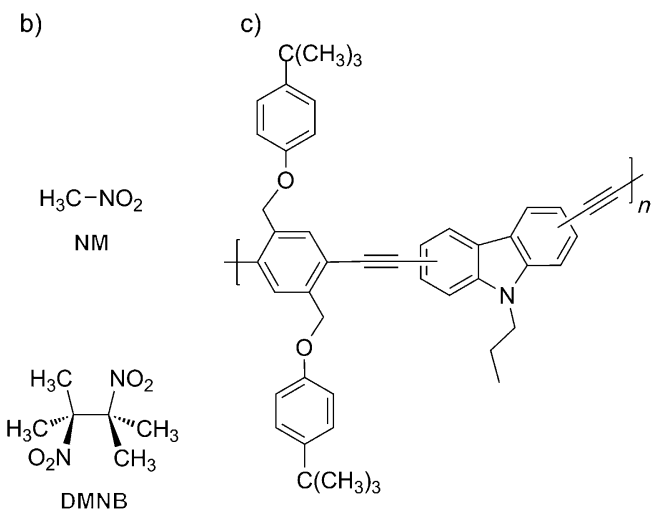

TBP-PPE-3,6-CBZ: 3,6-linkage on CBZ TBP-PPE-2,7-CBZ: 2,7-linkage on CBZ

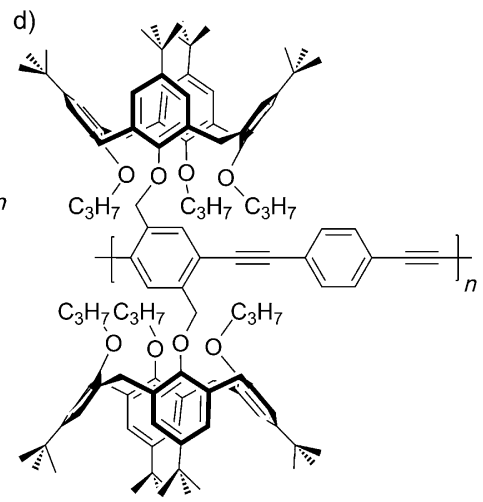

CALIX-p-PPE

Scheme 1. Molecular structures of a) poly(\{2,5-bis-[5,11,17,23-tetrakis(1,1-dimethylethyl)-25-(oxymethyl)-26,27,28-tripropyloxycalix[4]arene]-p-phenylene\}ethynylene-alt-3,6 or 2,7-carbazolyleneethynylene) (CALIX-PPE-CBZs); b) NM and DMNB; c) poly[2,5-bis( $p$-tert-butylphenoxymethyl)-p-phenylene]ethynylene-alt-3,6 or 2,7-carbazolyleneethynylene (TBP-PPE-CBZs); and d) poly(\{2,5-bis-[5,11,17,23-tetrakis(1,1-dimethylethyl)-25-(oxymethyl)-26,27,28-tripropyloxycalix[4]arene]-pphenylene\}ethynylene-p-phenylene ethynylene) (CALIX-p-PPE). 
peating units of the host, the rigidity of the host and, in solution, by solvation effects. ${ }^{[31]}$ Complex formation between single calix[4]arene derivatives and NM have been reported in apolar media $\left(\mathrm{CHCl}_{3}\right.$ and $\left.\mathrm{CCl}_{4}\right)$ and in the solid state. ${ }^{[32]}$ The X-ray crystal structure of a calix[4]arene-bis-crown complex showed the formation of a 1:1 endo complex, in which the methyl group of NM entered deeply into the cavity of the calix[4]arene. In solution, $\mathrm{CH}-\pi$ interactions were deduced from NMR spectroscopy studies that showed an upfield shift of the $\mathrm{CH}_{3}-\mathrm{NO}_{2}$ resonance owing to ring-current effects of the aromatic rings. On the other hand, the only report we are aware of regarding calix[4]arene-DMNB interactions involved the use of a lower-rim amide-bridged $p$-tert-butylcalix[4]arene derivative/hydroxyl-terminated silicone oil coated fiber for solid-phase microextraction coupled to gas chromatography. The device showed enhanced extraction ability for DMNB relative to other commercial coatings. ${ }^{[33]}$

In the case of our recognition/signalizing systems (CALIXPPE-CBZs polymers), we believe that sequestered guests, either as endo- or exo-cavity complexes with the calixarene units, are held in close proximity $(<10 \AA)$ to the transduction centers located at the polymer backbone; this allows feasible electron transfer after photoinduction, and thus, leads to observed fluorescence quenching.

Figure 1 illustrates how PET may occur: after excitation, an electron is transferred from the singlet excited state of the fluorophore ('SOMO*) to the LUMO of the guest followed by nonradiative back electron transfer to the fluorophore, in which the excitation energy is dissipated by vibrational relaxation of the guest. In addition to this probable sphere-of-action mechanism (an apparent static quenching), ${ }^{[34]}$ the formation of encounter complexes between the guest and fluorophore should also take place and lead to collisional quenching.

The quenching efficiencies determined for thin films $(\approx 20 \mathrm{~nm})$ of CALIX-PPE-3,6-CBZ and CALIX-PPE-2,7-CBZ obtained by using NM as an analyte are shown in Figure 2 .

The first thing that should be noted is the remarkable sensitivity and fast response of both polymers for NM vapor. Indeed, after only $10 \mathrm{~s}$ of exposure to equilibrium vapor of NM (vapor pressure of $35.925 \mathrm{~mm} \mathrm{Hg}$ at $25^{\circ} \mathrm{C} ; 47270 \mathrm{ppm}$ ), ${ }^{[35]}$ more than $50 \%$ of fluorescence quenching was observed for CALIX-PPE-3,6-CBZ, which is the most sensitive material, and reached over $75 \%$ upon prolonged exposure (900 s). For
CALIX-PPE-2,7-CBZ, lower values (38 and $46 \%$ ) were obtained for the same periods.

Under the same experimental conditions, quenching by DMNB vapors (vapor pressure of $2.07 \times 10^{-3} \mathrm{~mm} \mathrm{Hg}$ at $25^{\circ} \mathrm{C}$; $2.7 \mathrm{ppm})^{[36]}$ was then evaluated (Figure 3). Again, an extraordinary sensitivity was demonstrated within only $10 \mathrm{~s}$ of exposure: $38 \%$ of fluorescence quenching for CALIX-PPE-3,6-CBZ, which remained almost the same (44\%) for extended periods (900 s). The results for CALIX-PPE-2,7-CBZ were moderate (ca. $18 \%)$.

The sensing abilities toward NM and DMNB were further assessed with a $p$-PPE main-chain polymer (CALIX-p-PPE; cf. Scheme 1d), which possessed a low-lying LUMO (see below). By using thin films of identical thicknesses, only sparing deviations from the initial luminescence intensities were found with either DMNB ( $a \approx 2 \%$ fluorescence increase upon exposure for $10 \mathrm{~s}$, which was maintained over $15 \mathrm{~min}$ ) or NM (a decrease of $6 \%$ at $10 \mathrm{~s}$, which evolved to $4 \%$ on $15 \mathrm{~min}$; see Figure $\mathrm{S} 4$ in the Supporting Information); this unequivocally showed the lack of sensing ability of this material toward NM and DMNB.

To obtain more knowledge about the distinct behavior presented by these polymers, we performed quantum mechanical calculations (B3LYP/6-311G*) ${ }^{[37]}$ on appropriate models that described the different polymer main chains, and on the two tested analytes (NM, DMNB). The location and shape of the LUMOs of the polymer models and those of the nitroaliphatic guests are depicted in Figure S5 in the Supporting Information.

Analysis of the energy correlation diagram (Figure 4) gives some valuable information on the propensity of thermodynamically driven electron-transfer processes. First, the LUMO energies of the CALIX-PPE-CBZs models lie within the range of LUMO energies of all analytes, even for NM, which has a highlying LUMO $(-2.06 \mathrm{eV})$, and provides an appropriate driving force for PET. Second, the model for CALIX-PPE-3,6-CBZ has the highest LUMO energy $(-1.58 \mathrm{eV})$, which means that a better driving force exists for electron transfer from the ${ }^{1} \mathrm{SOMO}^{*}$ of the polymer to the analyte LUMO; this is in excellent agreement with what was experimentally observed if one compared the performance of the two CALIX-PPE-CBZs. Third, the LUMO energy $(-2.16 \mathrm{eV})$ of the CALIX-p-PPE model clearly does not match the LUMO energy of NM; thus it should be expected that electron transfer from the singlet excited state SOMO
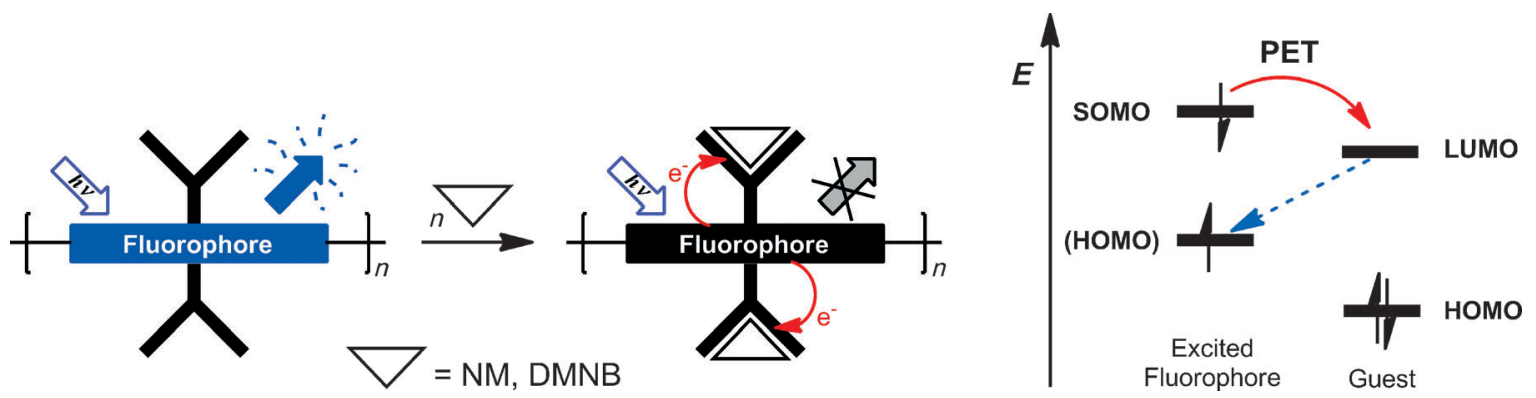

Figure 1. Left: A cartoon showing the fluorescence quenching of CALIX-PPE-CBZs by NM or DMNB. Right: A schematic frontier orbital energy diagram showing electron transfer from the 'SOMO* of the excited fluorophore host to the LUMO of the guest, followed by back electron transfer to the fluorophore. 

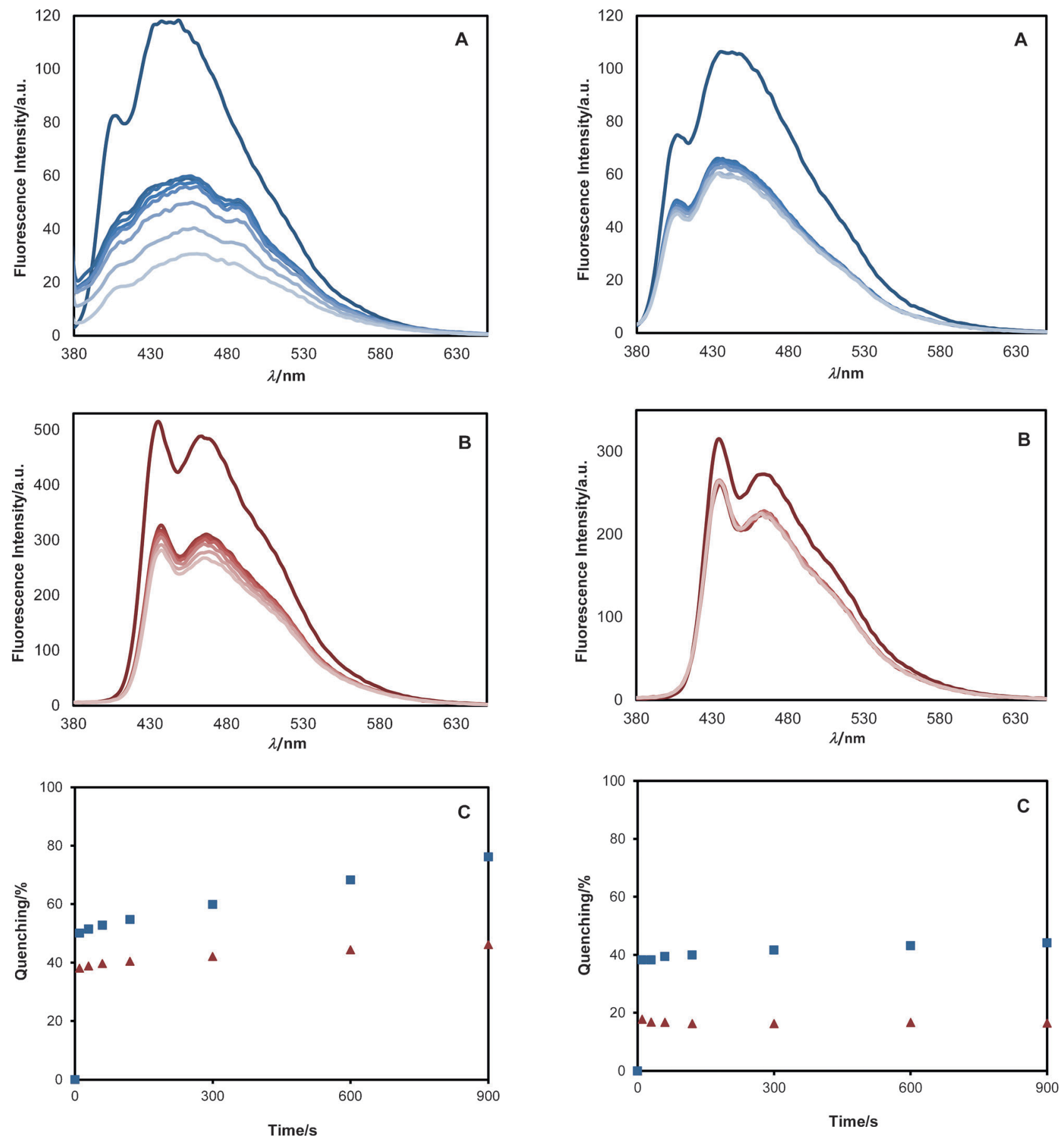

Figure 2. Time-dependent emission intensities of A) CALIX-PPE-3,6-CBZ and B) CALIX-PPE-2,7-CBZ thin films $(\approx 20 \mathrm{~nm})$ after being exposed to saturated NM vapor $(47270 \mathrm{ppm})$ at $25^{\circ} \mathrm{C}(0,10,30,60,90,120,300,600$, and $900 \mathrm{~s}$, from top to bottom), and the corresponding fluorescence quenching efficiencies (C) for both materials ( $\boldsymbol{\square}$ from $A, \Delta$ from $B ; \lambda_{\text {exc }}=360 \mathrm{~nm}$ ).

should not occur and explained the experimentally observed absence of quenching. In the case of DMNB, and in spite of the slightly favorable driving force for electron transfer, no quenching took place; this was likely to be due to the absence of reasonable fluorophore-analyte binding interactions.

From the above experimental results and frontier orbital energy correlations, it could be safely stated that, first of all,

Figure 3. Time-dependent emission intensities of A) CALIX-PPE-3,6-CBZ and B) CALIX-PPE-2,7-CBZ thin films $(\approx 20 \mathrm{~nm})$ after being exposed to saturated DMNB vapor $(2.7 \mathrm{ppm})$ at $25^{\circ} \mathrm{C}(0,10,30,60,90,120,300,600$, and $900 \mathrm{~s}$, from top to bottom), and the corresponding fluorescence quenching efficiencies (C) for both materials ( $\boldsymbol{\|}$ from $A, \boldsymbol{\Delta}$ from $B ; \lambda_{\text {exc }}=360 \mathrm{~nm}$ ).

a suitable driving force for electron transfer from the "SOMO* of the polymer to the analyte LUMO should exist, otherwise no quenching activity would be observed. However, this single thermodynamic condition is far from being the only parameter that would dictate and assure high quenching efficiencies of a given fluorophore, and thus, prompt its use as a chemical sensor. Indeed, the fluorescence quenching of thin films by 

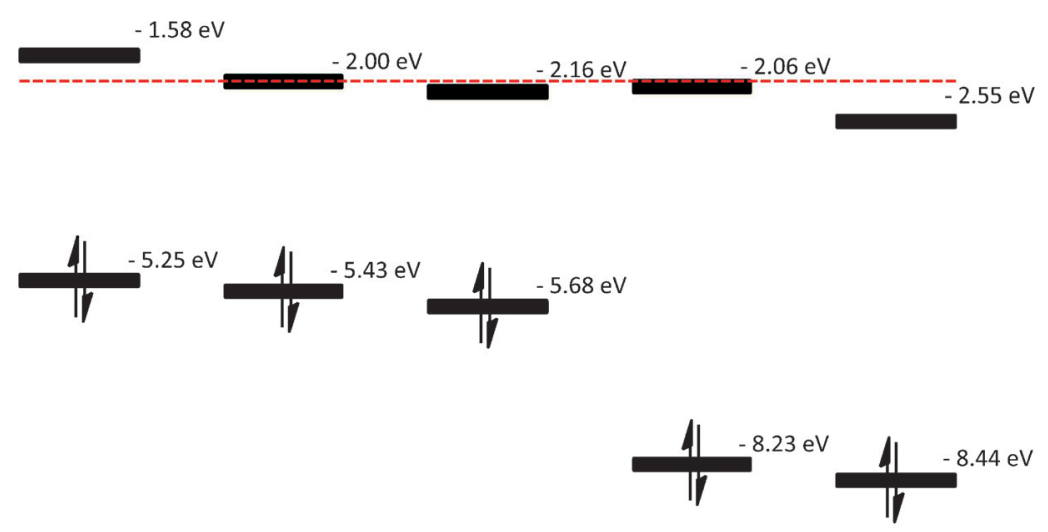

$\begin{array}{llll}\text { PPE-3,6-CBZ PPE-2,7-CBZ PPE model NMNB } & \text { NM }\end{array}$

Figure 4. HOMO and LUMO energy levels of the polymer main-chain models (PPE-3,6-CBZ, PPE-2,7-CBZ and PPE), and those of NM and DMNB. Values were determined from the results of ab initio calculations at the B3LYP/6$311 \mathrm{G}^{*}$ level of theory. ${ }^{[37]}$ tions for the spin-coating process. On the other hand, owing to the low solid-state quantum yield of TBP-PPE-CBZs (see the Supporting Information), higher thicknesses $(\approx 50 \mathrm{~nm})$ were used whenever possible (TBP-PPE-3,6CBZ) to retrieve reasonable signal-to-noise ratios in the measurements. The quenching efficiencies for both TBP-based materials are gathered in Figure 6.

The highest response to the analyte vapors came from TBPPPE-3,6-CBZ, which showed only modest fluorescence quenching vapors of nitroaromatic or nitroaliphatic compounds relies on several other parameters beyond the exergonicity of electron transfer, such as the strength of binding (electrostatic or other) between the electron-donor $\pi$ system and the electron acceptor, the vapor pressure of the analyte, and the film thickness. ${ }^{[38-39]}$

To gain a deeper understanding on how these several factors come into play, we first addressed the importance of the calixarene components as recognition elements in the overall efficiency of the sensory system. Toward this end, two new polymers (TBP-PPE-CBZs, Scheme 1c), which possessed the same main chains as those of CALIX-PPE-CBZs, but in which the bis-calixarene units were absent, were synthesized and their photophysical properties elucidated (see the Supporting Information). A representative time-dependent quenching plot for TBP-PPE-2,7-CBZ with NM is shown in Figure 5. TBP-PPE2,7-CBZ films with thicknesses of approximately $15 \mathrm{~nm}$ were used in the experiments because the low solubility of the material precluded the preparation of higher concentration solu-

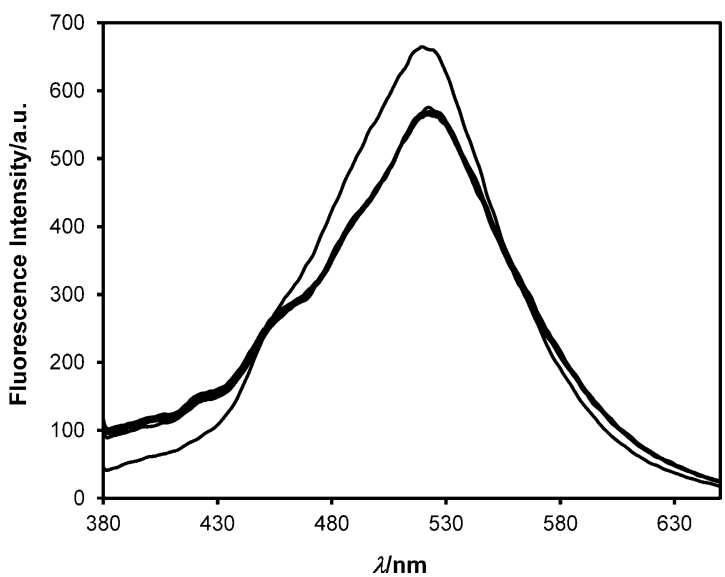

Figure 5. Time-dependent emission intensities of a TBP-PPE-2,7-CBZ thin film $(\approx 15 \mathrm{~nm})$ after being exposed to saturated $\mathrm{NM}$ at $25^{\circ} \mathrm{C}(0,10,30,60,90$, $120,300,600$, and $900 \mathrm{~s}$, from top to bottom; $\lambda_{\text {exc }}=360 \mathrm{~nm}$ ).

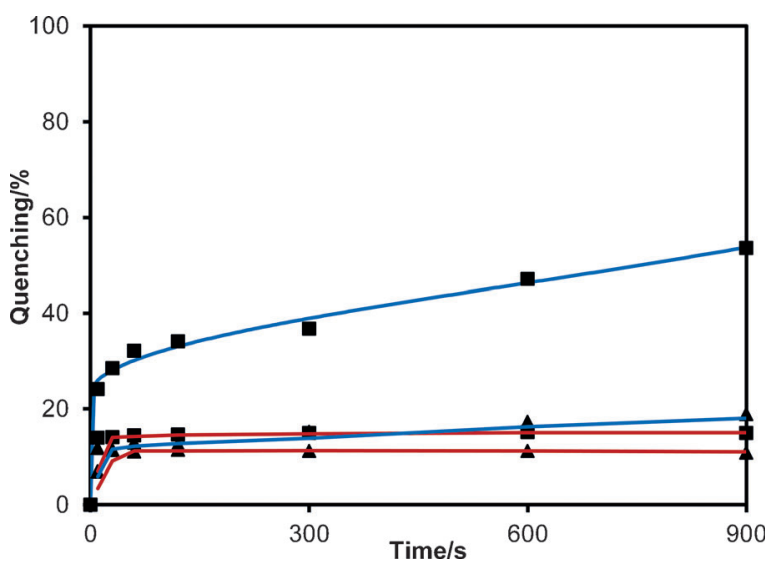

Figure 6. Fluorescence quenching efficiencies for thin films $(\approx 15$ and $50 \mathrm{~nm}$, respectively) of TBP-PPE-2,7-CBZ (red) and TBP-PPE-3,6-CBZ (blue) with NM (घ) and DMNB ( $\mathbf{\Lambda})$, after excitation at $\lambda=360 \mathrm{~nm}$.

efficiencies for NM and DMNB (24 and $12 \%$, respectively, after $10 \mathrm{~s}$ of exposure). Differences in the quenching efficiency of the two TBP polymers may be justified by using the same principles enunciated before for CALIX-PPE-CBZs (because they have identical main chains), and could also be traced to the larger film thickness used in the experiments with TBP-PPE-3,6CBZ (see below). If these results are compared with those of CALIX-PPE-CBZs, one clearly identifies the unequivocal superior performance of the calix[4]arene-based polymers as sensor materials for NM and DMNB (when using films with similar thicknesses; see below).

The influence of the film thickness on the quenching efficiency was next accessed for the CALIX-PPE-CBZs polymers with both analytes (Figure 7). The limited solubility and/or the poor film quantum efficiency of TBP-PPE-CBZs prevented the study of such an effect in these polymers. The data show a noticeable sensitivity enhancement for both polymers toward NM and DMNB on going from the thinnest $(\approx 2 \mathrm{~nm}$ ) to the thickest films $(\approx 40 \mathrm{~nm}){ }^{[40]}$ If the behavior of the two analytes are com- 


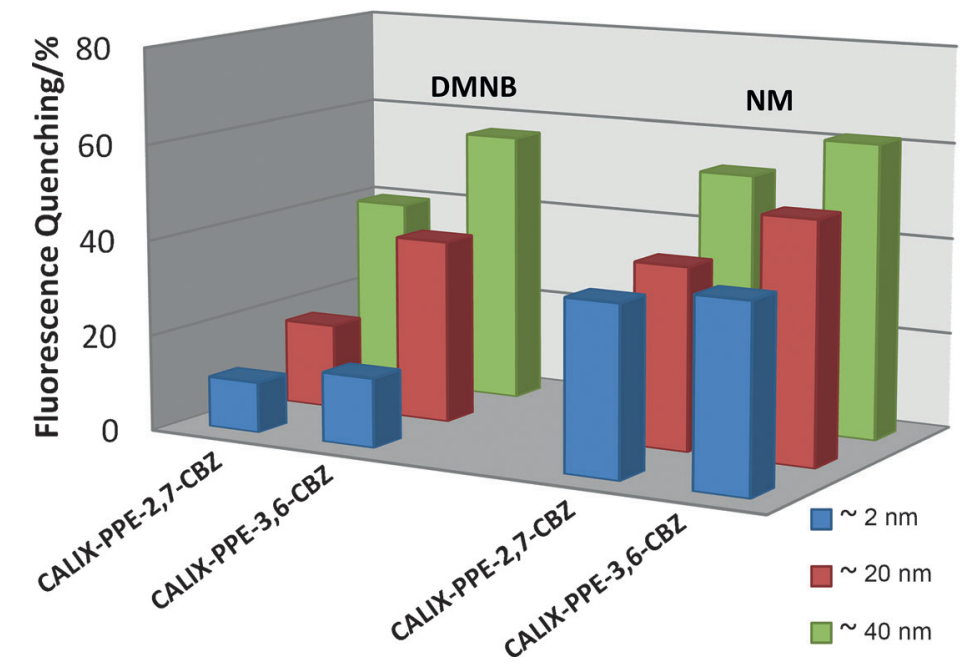

Figure 7. Fluorescence quenching responses of thin films of CALIX-PPE-CBZs with different thicknesses $(\approx 2,20$, and $40 \mathrm{~nm})$ upon exposure to saturated vapors of NM and DMNB for $10 \mathrm{~s}$ at $25^{\circ} \mathrm{C}$.

pared, the data seem to suggest that the reduced vapor pressure of DMNB (compared to NM) is considerably outweighed by its higher electron affinity, which helps to explain the not too dissimilar quenching efficiencies, in particular, if thicker films are used.

Overall, and under the conditions tested, CALIX-PPE-3,6-CBZ is the best sensory material for NM and DMNB and reaches around $60 \%$ after only $10 \mathrm{~s}$ of exposure. ${ }^{[41]}$

Having established the underlying key features of the sensing abilities of CALIX-PPE-CBZs toward NM and DMNB, we screened two other compounds (DMF and benzene) ${ }_{1}^{[24]}$ which possessed different electronic properties and vapor pressures, ${ }^{[42]}$ to evaluate the selectivity of our materials. By using the same experimental setup (FF illumination, $\lambda_{\text {exc }}=360 \mathrm{~nm}$ ), only a negligible fluorescence attenuation $(\approx 1 \%)$ was found after 1 min of exposure to DMF vapor, whereas an increase in luminescence intensity was initially registered for benzene, which progressively returned to the original intensity that was restored after about $15 \mathrm{~min}$. These results, which are in contrast to those of other reported sensing materials, ${ }^{[24]}$ further highlight the excellent performance attained in the detection of NM and DMNB by our CALIX-PPE-CBZs polymers. Indeed, in addition to the high sensitivity already shown, notable selectivity is also achieved.

The photostability of each polymer was evaluated under the same setup and illumination conditions of the quenching experiments $\left(\lambda_{\text {exc }}=360 \mathrm{~nm}\right.$; air-equilibrated samples in a sealed cell). For all polymers, high photostability was found. Only slight photobleaching was observed for CALIX-PPE-3,6-CBZ and TBP-PPE-3,6-CBZ upon prolonged exposure (up to $9 \%$ after $10 \mathrm{~min})$. For CALIX-PPE-2,7-CBZ, no photodegradation could be accurately measured $(<1.5 \%$ decrease in fluorescence intensity after $10 \mathrm{~min}$; see Figure $\mathrm{S} 8$ in the Supporting Information).

The reversibility of the quenching processes was assessed through a series of continuous fluorescence quenching-recov- ery tests. Upon exposure to NM or DMNB vapors, the emission intensities of the CALIX-PPE-CBZ films quickly recovered after being exposed to a flow of nitrogen for $15 \mathrm{~min}$ (Figure S9 in the Supporting Information). If the healed films were re-exposed to DMNB or NM vapors, similar quenching activity registered; this suggested the reusability of the films in practical sensor devices.

\section{Conclusion}

Through the use of two calix[4]arene-based CPs (CALIX-PPE-CBZs), we demonstrated the cooperativity of such systems in signalizing the presence of a nitroaliphatic liquid explosive (NM) and an explosive taggant (DMNB) in the vapor phase with exceptional sensitivity and selectivity in a fast and reversible manner. Ab initio calculations performed on model polymers with different isomeric carbazole repeating units (3,6- and 2,7-connectivities) provided clear-cut evidence for the most favorable thermodynamically driven PET, which clearly favored CALIX-PPE-3,6-CBZ. To prove that an exergonic electron transfer was not the only intervening parameter that dictated the superior sensing ability of CALIX-PPE-CBZ polymers toward these analytes (e.g., in comparison to CALIX-p-PPE), fluorescence quenching efficiencies were also determined for two newly synthesized TBP-PPE-CBZ polymers, in which the bis-calixarene units were absent, but otherwise the main chains were similar. Although active in the detection of NM and DMNB, much better performance was achieved with CALIX-PPE-CBZs; this fully corroborated our working hypotheses that the calixarene hosts were the unique differentiating element responsible for overall enhanced sensing in the solid state.

\section{Experimental Section}

\section{Instruments and methods}

Steady-state fluorescence spectra were collected on a Perkin-Elmer LS45 fluorimeter by using a $1 \mathrm{~cm}$ quartz cuvette in front-face (FF) geometry (oriented at $\mathrm{ca} .30^{\circ}$ relative to the incident beam) at $25^{\circ} \mathrm{C}$ in air-equilibrated conditions. The evaluation of the solidstate photophysical properties (see Table S1 in the Supporting Information) and quenching efficiencies were determined in thin films prepared by spin-coating (home-made spin-coater; spin-rate of $1500 \mathrm{rpm}$ ); films with different thicknesses were obtained from solutions of appropriate concentrations in chloroform on quartz substrates $(4 \times 0.8 \mathrm{~cm})$, which were dried in vacuo for $1 \mathrm{~h}$ before use. The fluorescence was measured by using FF illumination. The average thickness of spin-casted films was determined with a Veeco Dektak 6M profilometer. Films with optical densities (ODs) ranging from 0.06 to 0.23 at the excitation wavelength $(\lambda=$ $360 \mathrm{~nm}$ ) were used to set up a calibration curve. A linear relationship $\left(R^{2}=0.9971\right)$ between the optical data and film thicknesses was observed within this range. For $\mathrm{ODs}<0.06$, the corresponding thicknesses were estimated by data extrapolation. Fluorescence quenching experiments with thin films were performed as follows: The film was introduced into a cell, a spectrum was recorded (ini- 
tial fluorescence, $F_{0}$ ), and the film was removed. A given analyte was then dispensed into the same cuvette, covered with cotton gauze, and the cell was sealed and kept at $25^{\circ} \mathrm{C}$ for $16 \mathrm{~h}$ to allow for vapor-pressure equilibration. The thin film was then carefully introduced into the cell and the spectra were acquired over time, keeping the cell sealed. The quenching efficiencies $\left(\left(F_{0}-F\right) / F_{0} \times 100\right)$ were computed with the data obtained in the absence of the analyte.

Geometry optimizations and energy calculations were performed with a hybrid density functional model (B3LYP) using $6-311 \mathrm{G}^{*}$ basis sets, as developed in the Spartan'04 molecular modeling program. ${ }^{[37]}$ For polymer models, equilibrium geometries were first optimized by means of the AM1 semi-empirical model, ${ }^{[37]}$ followed by single-point energy calculations by using DFT/B3LYP/6-311G*.

\section{Materials}

CALIX-PPE-3,6-CBZ, ${ }^{[29]}$ CALIX-PPE-2,7-CBZ, ${ }^{[29]}$ and CALIX-p-PPE ${ }^{[30]}$ were synthesized according to our reported methods. The number-average molecular weights and degrees of polymerization of the polymer batches used in this study were of similar magnitude to those presented in the original reports, as estimated by ${ }^{1} \mathrm{H}$ NMR spectroscopic end-group analysis. The syntheses of TBPPPE-3,6-CBZ and TBP-PPE-2,7-CBZ, as well as their structural and photophysical characterization, are described in the Supporting Information. NM (98\%, Aldrich) and DMNB (98\%, Aldrich) were used as received.

\section{Acknowledgements}

We thank the Fundação para a Ciência e a Tecnologia/MCTES (Portugal) for partial financial support (PEst-OE/EQB/UI0702/ 2011). We also thank Prof. Manuel J. Matos (Instituto Superior de Engenharia de Lisboa and Instituto de Telecomunicações) for his assistance with the profilometry measurements.

Keywords: calixarenes - carbazoles - explosives · host-guest systems $\cdot$ polymers $\cdot$ fluorescence

[1] Y. Salinas, R. Martínez-Máñez, M. D. Marcos, F. Sancenón, A. M. Costero, M. Parra, S. Gil, Chem. Soc. Rev. 2012, 41, 1261-1296.

[2] M. E. Germain, M. J. Knapp, Chem. Soc. Rev. 2009, 38, 2543-2555.

[3] M. S. Meaney, V. L. McGuffin, Anal. Bioanal. Chem. 2008, 391, $2557-$ 2576.

[4] S. W. Thomas III, G. D. Joly, T. M. Swager, Chem. Rev. 2007, 107, 13391386.

[5] S. J. Toal, W. C. Trogler, J. Mater. Chem. 2006, 16, 2871-2883.

[6] J. D. Sullivan, W. B. Sunderland, Liquid Explosive in Pipes, U.S. Army Research Laboratory Report, 1994.

[7] H. Shekhar, Cent. Eur. J. Energ. Mater. 2012, 9, 87-97.

[8] For a survey of the patent literature describing explosive compositions comprising NM and a sensitizer, see ref. [7].

[9] Allegedly, bombings of the Murrah Federal Office Building in Oklahoma City, USA, 1995 and Government buildings in Oslo, Norway, 2011.

[10] J. V. Goodpaster, V. L. McGuffin, Anal. Chem. 2001, 73, 2004-2011.

[11] K.-S. Focsaneanu, J. C. Scaiano, Photochem. Photobiol. Sci. 2005, 4, $817-$ 821.

[12] M. S. Meaney, V. L. McGuffin, Anal. Chim. Acta 2008, 610, 57-67.

[13] H. Wang, Y. Fang, L. Ding, L. Gao, D. Hu, Thin Solid Films 2003, 440, $255-260$.

[14] J. Kang, L. Ding, F. Lü, S. Zhang, Y. Fang, J. Phys. D 2006, 39, $5097-$ 5102.
[15] K. J. Balkus, Jr., T. J. Pisklak, G. Hundt, J. Sibert, Y. Zhang, Microporous Mesoporous Mater. 2008, 112, 1-13.

[16] Y. Z. Liao, V. Strong, Y. Wang, X.-G. Li, X. Wang, R. B. Kaner, Adv. Funct. Mater. 2012, 22, 726-735.

[17] C. Zhang, Y. Che, X. Yang, B. R. Bunes, L. Zang, Chem. Commun. 2010, 46, 5560-5562.

[18] S. Pramanik, C. Zheng, X. Zhang, T. J. Emge, J. Li, J. Am. Chem. Soc. 2011, 133, 4153-4155.

[19] A. J. Clulow, P. L. Burn, P. Meredith, P. E. Shaw, J. Mater. Chem. 2012, 22, $12507-12516$.

[20] Containing the Threat from Illegal Bombings: An Integrated National Strategy for Marking, Tagging, Rendering Inert, and Licensing Explosives and Their Precursors, The National Academies Press, Washington, DC, 1998, p. 49

[21] Convention on the Marking of Plastic Explosives for the Purpose of Detection, United Nations Council of the International Civil Aviation Organization, March 1, 1991; ref. [20].

[22] S. W. Thomas III, J. P. Amara, R. E. Bjork, T. M. Swager, Chem. Commun. 2005, 4572-4574.

[23] T. Naddo, X. Yang, J. S. Moore, L. Zang, Sens. Actuators B 2008, 134, $287-291$.

[24] A. Lan, K. Li, H. Wu, D. H. Olson, T. J. Emge, W. Ki, M. Hong, J. Li, Angew. Chem. 2009, 121, 2370-2374; Angew. Chem. Int. Ed. 2009, 48, $2334-$ 2338.

[25] G. Tang, S. S. Y. Chen, P. E. Shaw, K. Hegedus, X. Wang, P. L. Burn, P. Meredith, Polym. Chem. 2011, 2, 2360-2368.

[26] B. P. Morgan, R. J. Gilliard, Jr., R. S. Loungani, R. C. Smith, Macromol. Rapid Commun. 2009, 30, 1399-1405.

[27] M. E. Germain, T. R. Vargo, P. G. Khalifah, M. J. Knapp, Inorg. Chem. 2007, $46,4422-4429$.

[28] D. A. Olley, E. J. Wren, G. Vamvounis, M. J. Fernee, X. Wang, P. L. Burn, P. Meredith, P. E. Shaw, Chem. Mater. 2011, 23, 789-794.

[29] P. D. Barata, A. I. Costa, J. V. Prata, React. Funct. Polym. 2012, 72, 627634.

[30] A. I. Costa, L. F. V. Ferreira, J. V. Prata, J. Polym. Sci. Part A 2008, 46, $6477-6488$.

[31] A. Arduini, A. Pochini, A. Secchi, F. Ugozzoli in Calixarenes 2001 (Eds.: Z. Asfari, V. Böhmer, J. Harrowfield, J. Vicens), Kluwer Academic Publishers, Dordrecht, 2001, pp. 457-475.

[32] A. Arduini, W. M. McGregor, D. Paganuzzi, A. Pochini, A. Secchi, F. Ugozzoli, R. Ungaro, J. Chem. Soc. Perkin Trans. 2 1996, 839-846.

[33] X. Li, Z. Zeng, Y. Zeng, Talanta 2007, 72, 1581-1585.

[34] J. R. Lakowicz, Principles of Fluorescence Spectroscopy, 3rd ed., Springer, New York, 2006, pp. 277-286.

[35] Handbook of Chemistry and Physics, 84th ed. (Ed.: D. R. Lide), CRC Press, Boca Raton, 2004, chap. 15, p. 17.

[36] See Reference [20], p. 47.

[37] Spartan'04 Molecular Modeling Program, Wavefunction, Inc., Irvine, CA, 2005.

[38] J.-S. Yang, T. M. Swager, J. Am. Chem. Soc. 1998, 120, 11864-11873.

[39] Z. Wang, Z. Y. Wang, J. Ma, W. J. Bock, D. Ma, Polymer 2010, 51, 842 847.

[40] The present data aims to establish a trend in the sensitivity performance in relation to the thicknesses of the thin films, and by no means should the latter be considered as optimized.

[41] Solution quenching experiments also showed that CALIX-PPE-3,6-CBZ displayed higher quenching constants than those of CALIX-PPE-2,7-CBZ (albeit low) for NM and DMNB. Under these conditions, however, DMNB was a more effective quencher than that of NM. For a detailed description of these data, see the Supporting Information.

[42] LUMO energies calculated at the B3LYP/6-311G* level:: ${ }^{[37]} E_{\text {LUMO(DMF) }}=+$ $0.62 \mathrm{eV} ; E_{\mathrm{LUMO} \text { (benzene) }}=-0.22 \mathrm{eV}$. Dipole moments and vapor pressures (at $25^{\circ} \mathrm{C}$ ) were taken from reference [35]: DMF: $\mu=3.82 \mathrm{D}, 3.3 \mathrm{~mm} \mathrm{Hg}$; benzene: $\mu=0 \mathrm{D}, 95.25 \mathrm{~mm} \mathrm{Hg}$.

Received: August 1, 2013

Published online on October 11, 2013 
Copyright of ChemPlusChem is the property of Wiley-Blackwell and its content may not be copied or emailed to multiple sites or posted to a listserv without the copyright holder's express written permission. However, users may print, download, or email articles for individual use. 